University of Nebraska - Lincoln

DigitalCommons@University of Nebraska - Lincoln

Robert Katz Publications

Research Papers in Physics and Astronomy

September 1982

\title{
Thresholds in Radiobiology
}

Robert Katz

University of Nebraska-Lincoln, rkatz2@unl.edu

Werner Hofmann

University of Salzburg, Austria

Follow this and additional works at: https://digitalcommons.unl.edu/physicskatz

Part of the Physics Commons

Katz, Robert and Hofmann, Werner, "Thresholds in Radiobiology" (1982). Robert Katz Publications. 114. https://digitalcommons.unl.edu/physicskatz/114

This Article is brought to you for free and open access by the Research Papers in Physics and Astronomy at DigitalCommons@University of Nebraska - Lincoln. It has been accepted for inclusion in Robert Katz Publications by an authorized administrator of DigitalCommons@University of Nebraska - Lincoln. 
Published in Physics in Medicine and Biology 27:9 (September 1982), pp. 1187-1190. Copyright (C) 1982

Institute of Physics Publishing. Used by permission. http://www.iop.org/EJ/journal/PMB

LETTERS TO THE EDITOR

\section{Thresholds in Radiobiology}

The Editor,

Sir,

Interpretations of radiation effects frequently call upon the word "threshold." In this letter we wish to explore the meaning of this word, its relationship to the fundamental character of radiation effects, and to the question of perception. We make no distinction between "stochastic effects" and "non-stochastic effects" (ICRU 1971, ICRP 1977). As conceived here, all interactions of radiation with matter are probabilistic. At the cellular or molecular level, where most radiation effects originate, the statistical nature of ionization and excitation events gives rise to considerable fluctuation in the number of these primary events in sensitive sites. Whatever the array of primary events required in a sensitive site to initiate an observed end-point, the statistical character of these events argues against the existence of a threshold. In this sense, there are no non-stochastic processes. The severity of an effect in tissue is a measure of the probability of occurrence of the initiating event in the cells making up that tissue. There may be, however, a quasi-threshold of perception, associated with the emergence of detected events related to the radiation field above a background of events unrelated to that field. In particular, we propose that such questions as the possible existence of a low-dose threshold for cancer induction, or of a low-LET threshold for the activation of a sensitive target, must, a priori, be answered in the negative.

Inferences of threshold are common in radiation effects in all fields, perhaps suggested by the existence of energy levels. While there are energy thresholds for the transitions between atomic levels, and through the band gap in solids, within an order of magnitude or less of $10 \mathrm{eV}$, these energy thresholds play only a small role in the interaction of much more energetic charged particles with matter, where a free electron approximation is adequate for the description of the process. There are suggestions of a dose threshold in the study of electron resists used in the construction of micro-electronic wafers (Bowden 1979), just as there are suggestions of dose or LET thresholds in radiobiology (Bond 1971, Burch and Chesters 1981, Goodhead 1982). In the manufacturers' literature describing the properties of nuclear emulsions, there are suggestions of LET thresholds in emulsion response. Some emulsions are described as sensitive to singly charged particles of energy less than some critical value (Ilford 1968). So also primary ionization thresholds or restricted energy loss thresholds have been suggested for the production of etchable tracks in plastics (Fleischer et al. 1975).

If we are dealing with large numbers of ionizing particles, the statistical fluctuations may not be very significant. Thus we can expect that the probability distribution for the quantity of interest can be adequately approximated by the expectation value. The absorbed dose is a reasonable quantity at sufficiently high doses, whereas it is completely inappropriate at very low doses. But even where the number of participating particles is 
very high, as in thermodynamics, we are aware that probabilistic processes do not have thresholds, that there are still fluctuations, even in phase transitions.

When the number of detected events becomes very small these statistical fluctuations dominate the response of the system to the interacting agent. Carcinogenesis is such a case in point. Although final evidence about the mechanisms involved in radiation-induced carcinogenesis is still lacking, apart from classifying them as initiation and promotion events, they are nevertheless stochastic processes. If therefore the transformation of a single cell in an irradiated tissue can lead to carcinogenesis, it seems completely inappropriate to speak of a dose threshold however low the dose. This has already been pointed out by Mole (1975) for leukemia incidence in Japanese bomb survivors. The extreme rarity of malignant transformation per cell, however, may lead to the false impression of the existence of a dose threshold if the number of cancer cases is relatively small.

At low doses the use of absorbed dose is conceptually inappropriate because the irradiation may consist of well isolated particle paths. The dose-effect curve represents, in fact, a fluence versus incidence relationship. Although the probability of a cellular hit decreases with decreasing particle fluence, there cannot be a fluence threshold. Even multiple traversals cannot be excluded at low fluence. The question is one of positive identification, of observability, rather than one of threshold.

Another quasi-threshold of perception can be found in epidemiological data, e.g., cancer incidence in exposed population groups. Many factors, such as natural exposure, chemicals, diet, smoking habits, or genetic constitution may be responsible for the natural incidence rate, showing also for these reasons considerable variations between different population groups. Here the problem is the emergence of an effect from a background level where both the radiation induced cancer cases as well as the natural cases exhibit large statistical fluctuations, particularly at very low doses. Thus the exposure level at which the cancer probability exceeds background is sometimes mistakenly identified as a threshold exposure or dose. This quasi-threshold is much more pronounced in the case of a many-hit process - where we have a steeper dose-response curve, emerging at higher doses - than for a single hit mechanism. Since we suggest that cancer induction is a many hit process (Katz and Hofmann 1982), we would not be surprised if such quasi-thresholds were observed in radiation carcinogenesis.

Attempts to interpret the relationships between RBE and LET found for biological systems have engendered the suggestion that there are LET thresholds for different biological targets. Other investigators have found it tempting to accept the view that "targets" require well defined amounts of "energy," and that this energy is deposited in the passage of a particle of appropriate stopping power in a distance determined by the target size.

Just as in the case of "dose thresholds," which we rule out from a priori statistical considerations, we must also rule out LET thresholds from the same considerations. The interactions which arise from the passage of a high-LET particle are principally from secondary electrons (delta rays) whose generation is statistical. There is now a double statistical consideration, for the generation of the delta rays, and for the interaction of these delta rays with the targets. Thus from a priori statistical considerations, the concept of an LET threshold must be rejected.

Nuclear track emulsions provide experimental evidence for the validity of our conclusions. The formation of a latent image in an emulsion grain is also a stochastic process. To illustrate our point we have chosen to display the track of an energetic argon ion from the Berkeley BEVALAC accelerator in Ilford K-3 nuclear emulsion, which simulates radiobiological response (Katz 1981). In the top half of Figure 1 we display two strips of track pho- 


\section{${ }^{40} \mathrm{Ar}$ K-3 $100 \mu \mathrm{m}$ plate}
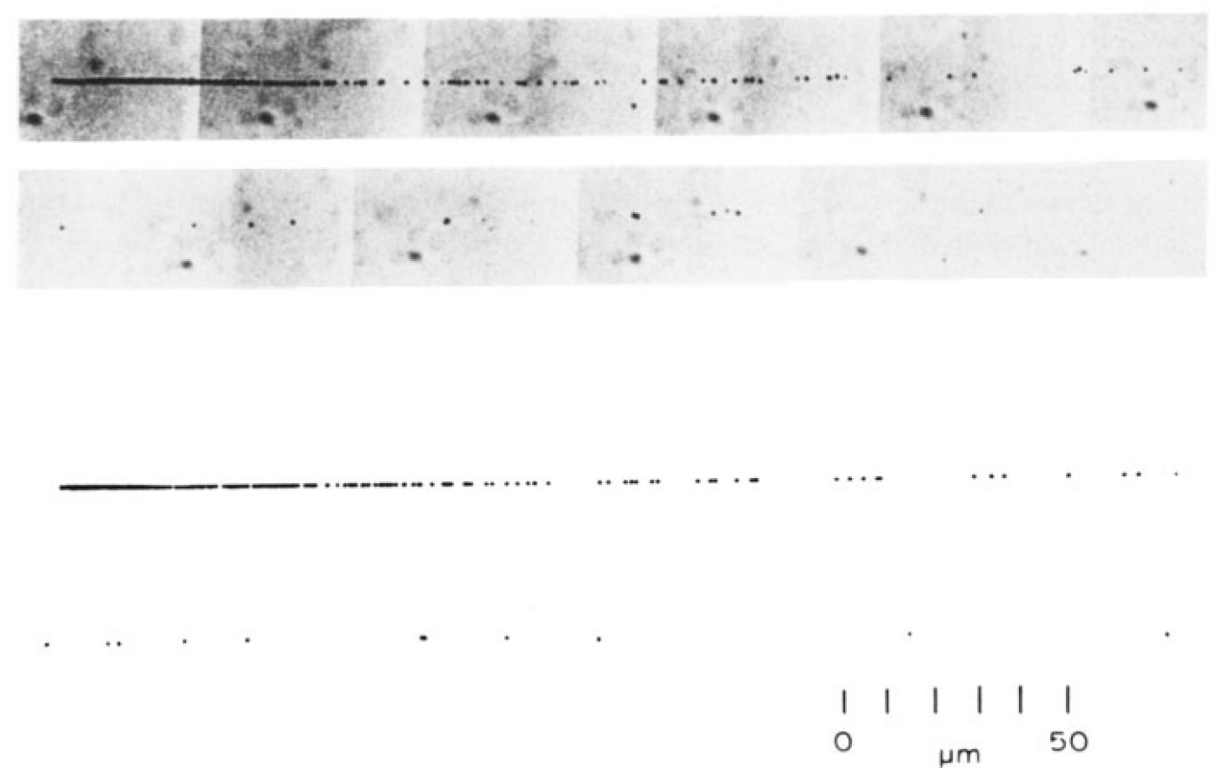

${ }^{40} \operatorname{Ar} C=5 E_{0}=2.3 \mathrm{~J} \mathrm{~cm}^{-3}$

Figure 1. The stopping $500 \mu \mathrm{m}$ of the track of a ${ }^{40} \mathrm{Ar}$ nucleus in an Ilford K-3 nuclear track plate coated to a thickness of $100 \mu \mathrm{m}$. The top pair of strips are a photomontage from an exposed emulsion while the lower pair of strips are from a computer simulation of the experimental photographs made by a trial and error assumption of the properties of the emulsion as a 5-or-more hit detector with $E_{0}=2.3 \mathrm{~J} \mathrm{~cm}^{-3}$. The motion of the particle is from right to left, and from bottom to top, stopping at the topmost left hand end.

tographs which together are the stopping $500 \mu \mathrm{m}$ of such a track. In the bottom half of this figure we display a computer generated track fitted to the real track by the assumption that this emulsion is a 5-or-more hit detector for which $E_{0}=2.3 \mathrm{~J} \mathrm{~cm}^{-3}$ (in emulsion). The stopping power increases from 3,700 to $14,000 \mathrm{MeV} \mathrm{g}^{-1} \mathrm{~cm}^{2}$ from a residual range of $500 \mu \mathrm{m}$ to the Bragg peak at $13 \mu \mathrm{m}$, respectively. The emulsion is so desensitized that the passage of a single electron is not observable as a track. The computer simulation assumes that the response of the emulsion to gamma rays is well described by the cumulative Poisson distribution for $C$-or-more hits with $C=5$. Thus the simulation assumes no dose threshold. With this dose-response function the calculated radial distribution of "local dose" from ejected delta rays is converted into a radial distribution of probability for grain activation. This is appropriately projected onto a plane to give the appearance of the particle track. Where the probability is small, grains are randomly located in the appropriate path length interval. Neither in the track photograph nor in the computer simulation is there any evidence of an LET threshold. We can imagine that we might gain the impression of a threshold if we were to impose a suitable density of randomly placed background grains.

We can see no conceptual difference between the interactions of radiation with emulsion grains and with the targets of biological systems. As in all cases the interactions of ra- 
diation with matter are statistical in character. Biological models cannot ignore the lessons from nuclear emulsions, where particle tracks can be observed, to create a scenario that is persuasive in a circumstance that is not subject to direct experimental verification, as in the case of tracks made up of killed or transformed cells in tissue (Katz and Hofmann 1982).

Once again we emphasize that although the existence of either a dose or a LET threshold can never be settled by experimental radiobiological investigations, we argue on fundamental statistical grounds that for all statistical processes, and especially where the number of observed events is small, the concept of a threshold is logically invalid.

\title{
Acknowledgments
}

This work is supported by the U.S. Department of Energy and the Max Kade Foundation.

\author{
Robert Katz \\ University of Nebraska-Lincoln \\ Lincoln, Nebraska 68588-0111, USA \\ Werner Hofmann \\ University of Salzburg, \\ A-5020 Salzburg, Austria
}

May 24, 1982

\section{References}

Bond, V. P. 1971 in Biophysical Aspects of Radiation Quality - 1971, STI/PUB/286 (IAEA: Vienna) p. 389.

Bowden, M. J. 1979 CRC Critical Reviews in Solid State and Material Science 8: 223.

Burch, P. R. J., and Chesters, M. S. 1981 Phys. Med. Biol. 26: 997.

Committee on the Biological Effects of Ionizing Radiations (BEIR III) 1980 The Effects on Populations of Exposure to Low Levels of Ionizing Radiation: 1980 (Washington, DC: National Academy Press).

Fleischer, R. L., Price, P. B., and Walker, R. M. 1975 Nuclear Tracks in Solids-Principles and Applications (Berkeley: University of California Press).

Goodhead, D. T. Radiat. Res., to be published.

International Commission on Radiological Protection 1977 ICRP Publication 26, Annals of the ICRP, vol. 1 , no. 3 .

ICRU 1971 Radiation Quantities and Units Report 19 (ICRU Publications, PO Box 30165, Washington, DC 20014, USA).

Ilford 1968 Technical Information Sheet Y 44.1.

Katz, R. 1981 in Proc. 7th Symp. on Microdosimetry, ed. J. Booz, H. G. Ebert, and H. D. Hartfiel (London: Harwood Academic for CEC), p. 831.

Katz, R., and Hofmann, W. 1982 Nucl. Instrum. Methods, to be published.

Mole, R. H. 1975 Br. J. Radiology 48: 157. 